\title{
Quantum Mechanical Postulates towards Realization of Artificial Wisdom on Incorporating Desire in Intelligence: A Hypothetical Study
}

\author{
Aloke Sarkar \\ Blast Furnace (Electrical), Rourkela Steel Plant, Steel Authority of India Limited, Rourkela, India \\ Email: alokesarkar@hotmail.com
}

How to cite this paper: Sarkar, A. (2021) Quantum Mechanical Postulates towards Realization of Artificial Wisdom on Incorporating Desire in Intelligence: A Hypothetical Study. Journal of Computer and Communications, 9, 63-79.

https://doi.org/10.4236/jcc.2021.912005

Received: November 23, 2021

Accepted: December 28, 2021

Published: December 31, 2021

Copyright $\odot 2021$ by author(s) and Scientific Research Publishing Inc. This work is licensed under the Creative Commons Attribution International License (CC BY 4.0).

http://creativecommons.org/licenses/by/4.0/

(c) (i) Open Access

\begin{abstract}
This paper is a hypothetical study to present postulates to have mathematical formulation towards realization of artificial wisdom. On referring quantum mechanics $(\mathrm{QM})$ a mathematical foundation of wisdom is being defined to have the wisdom machine that can work like creative human. Creation may be defined as the desire to have new. Wisdom is differentiated from intelligence on adding desire. Desire is defined as something more than need on encompassing knowledge of self and unbiased reasoning. Each component of desire has an embedded part that is defined as hallucination need (defined as wandering of mind around unreal, acting with attachments, having deluded mind with egoism, performing as foolish follower). This research is viewing wisdom as the tool-kit to realize the "Survival of the fittest." That is wisdom allows developing own-self on desire base. QM allows probabilistic approximation of multiple interacting events. Human behavior and creativity is a product of multiple events occurring simultaneously and/or in cascade with mutual interferences. Quantum mechanical interpretation of human behavior may be possible on describing it with dual aspects of desire (conscious or the real need and unconscious or the hallucination need) and uncertainties in simultaneous measurement of them. This paper is presenting nine postulates. These postulates and more to develop in future research may make it possible to mathematically encode human wisdom of creativity, so that there may be artificial wisdom.
\end{abstract}

\section{Keywords}

Intelligence, Need, Hallucination Need, Desire, Wisdom, Quantum Mechanics 


\section{Introduction}

\subsection{Declaration}

1) This paper is a hypothetical study to present postulates to have mathematical formulation towards realization of artificial wisdom. This study is author's very own radical thoughts. So it has no prior references besides author's own works.

2) Unless a thing is defined by measurement, it has no place in theory. So, it should be realized that these postulates need to be run through rigorous integrated research.

3) Quantum Mechanics is the description of the behavior of the matter and light in all its details and in particular, of the happenings on an atomic scale. This paper is presenting the postulates, those (along with more to come with through future research and statistical analysis) that could imitate human creative nature in machine, using quantum mechanics and its associative probability theories.

4) References [1] [2] [3] [4] are used to deliberate all quantum mechanical aspects of this paper. So it is not mentioned in the text more than this declaration.

\subsection{Prologues}

"Either a theory of knowledge is a theory about the meaning of the word 'know' and semantically related epistemic terms, or it is a theory about how people come to know what they do. The latter is not part of philosophy at all, but rather that part of psychology called learning theory." [5] These theories are outcome of human wisdom. But what is about Intelligence?

Intelligence is pervading each and every sphere of the human civilization. Presently it is not only limited to human intelligence (HI) but also the artificial intelligence (AI). The AI is the manifestation of human wisdom (HW). But what is the wisdom? How could we differentiate between wisdom and intelligence? Is there possibility to have artificial wisdom (AW)? "AI encompasses a variety of technologies which model human learning and decision-making behaviors. As yet, none of these technologies exhibit even animal-like consciousness and all are far from transcending their programming to achieve the emergent properties of the beings they attempt to model. The current 'narrow' or 'weak' AI is, by itself, fundamentally a data analysis tool (i.e., a means to an end) that does nothing more or less than its programming instructs it to do. It has no values or goals of its own, it simply follows the values and pursues the goals provided to it by its programmers. We have only begun to consider artificial wisdom (AW) as an essential complement with the potential to make AI a better tool and eventually perhaps more than a tool (i.e. an end in itself). At least for now, however, AW must also be programmed and therefore similarly reflects only the wisdom of its programmers." [6]

I am working a long to have answers for these questions (a short video describing my works is available in Twitter [7]). With references to my earlier 
works [8] [9] [10] here I am putting forth wisdom as integration of intelligence with desire with some quantum mechanical (QM) postulates that may answer these questions in future. This research is viewing wisdom as the tool-kit to realize the "Survival of the fittest." That is AW develops own-self. More to explain, say an autonomous vehicle is programmed by the designer and may have self developing characteristics on need base. Whereas a human pilot learns driving on own desire base. In this paper I am postulating desire something more than need, and also defining the "hallucination need". These postulates are introducing possibility of intensive mathematical treatment in human psychology and epistemology. With this mathematical base it may be possible to have machine that can have human like wisdom. These postulates and further proceedings in AW require an integrated research.

Quantum mechanics (QM) allows probabilistic approximation of multiple interacting events. It first weighs events with amplitudes. These amplitudes are in complex mathematical domain. These amplitudes can be operated with interference among them. The probability of occurrence can be easily obtained from these amplitudes. Human behavior and creativity is a product of multiple events occurring simultaneously and/or in cascade with mutual interferences. QM may allow easy realization of these.

It is being described that wisdom should have ethics and normative issues that differentiated it from intelligence. In my view these ethical and normative issues should be viewed as comparative term. A terrorist has the ethics to kill, whereas a saint has the ethics to save the life. There is nothing specific to be ethical to have wisdom. Wisdom incorporates ethics in the form of desires. Parts of desires are to be defined as combination of conscious and unconscious mind. It should have two mutually exclusive but bounded sections compromising of real needs and hallucination need. These sections can be represented using quantum mechanics. This paper is structured as: 1) society knowledge need \& wisdom, 2) wisdom differentiated from intelligence, 3) quantum mechanical postulates, and 4) conclusion. Sections 1 and 2 are outcomes of my earlier papers. Section 3 is being introduced here as quantum mechanical possibilities to encode mathematically desire and consequently wisdom to have machine with wisdom. These postulates are to be developed more in future research as outline in conclusion.

\section{Society Knowledge Need \& Wisdom}

We the human beings are social elements. We organize ourselves in organizations-family, social, professional and so on. This organization is our arrangements in complex groups. These groups are also interconnected and interacting. One's membership in a group is either by self-need, or by other's need, or by consequences of membership in other groups, or combination of these. So to codify our wisdom the starting point should be the organization. In organizations we share our knowledge using wisdom as tool. Also we organize our needs as per our resources, capabilities, and core competencies. In this view, each indi- 
vidual is a firm, and can be observed as knowledge organization that needs efficient knowledge management to strategically reach needs.

Core competency is defined by [11] as: 1) collective learning to coordinate diverse skills and integration of multiple knowledge bases. 2) Communication, involvement and a deep commitment to working across organizational boundaries. 3) Core competency grows over time. 4) It is not mere vertical integration but a collective integration or else superposition of actors and their activities. Superposition is neither mere addition nor positioning one upon other. It is a simultaneous activity. The concept of superposition is critical in QM.

Organizations exist because of their ability to create value and acceptable outcomes for various groups of stakeholders, people who have an interest, claim, or stake in the organization, in what it does, and in how well it performs [12]. Stakeholders are motivated to participate in an organization if they receive inducements (rewards-money, power, status etc.) that exceed the value of the contributions (skills, knowledge and expertise) they are required to make. So an organization can be viewed as the integration of behaviour variability of stakeholders-suppliers, customers, employees, managers, government, unions, community and general public [13]. Through knowledge-based view [14] of the firm (the firm is seen primarily as a vehicle for creating, integrating, storing and applying knowledge) a knowledge strategy is built on knowledge-based SWOT analysis (strength, weakness, opportunities and threats). As per [15] business strategy should be viewed less as a quest for monopoly rents (the return to market power) and more as a quest for Ricardian rents (the returns to the resources which confer competitive advantage over and above the real costs of these resources). The competitive advantage comes from cost advantage (process technology, size of plant, access to low-cost inputs) and differential advantage (brands, product technology, marketing-distribution-service capabilities). Wisdom may now be called as integration of knowledge and need in a context free regime or in a broad context regime to have the Ricardian rents.

Strategy may be defined as "the match an individual makes between own internal resources like health, knowledge etc. and the opportunities and risks created by external environment."

The five forces that impinge on a firm's (an individual's) ability to earn profits in an industry (a society or a group) and therefore determine the attractiveness of participating in that industry (society or group) are 1) the bargaining power of customers (we are calling this as physical need), 2) the bargaining power of the suppliers (safety need), 3) the threat of new entrants (social need), 4) the threat of substitute products (esteem need), 5) the strength and nature of traditional rivalry among firms in the industry (self-actualization need).

Maslow 1943 [16] said, "It is quite true that man lives by bread alone-when there is no bread. But what happens to man's desires when there is plenty of bread and when his belly is chronically filled? At once other (and higher) needs emerges and these, rather than physiological hungers, dominate the organism. And when these in turn are satisfied, again new (and still higher) needs emerge 
and so on...the basic human needs are organized into hierarchy of relative pre-potency." We are accustomed with the Maslow's Need Hierarchy that starts from physiological need and ends at self-actualization need.

"Physiological Needs" are food, water, oxygen, and sleep.

"Safety Needs" are feeling of safe and secure.

"Social Needs" include needs to have friends, to be loved and accepted by others, and to belong in social relationships. "Esteem Needs" call for self-respect, approval of others, and have success.

"Self-Actualization Needs" are needs of self-fulfilment and going beyond own selfish need.

Maslow has grouped physiological, safety and social needs as "deficiency needs" and esteem and self-actualization as "growth needs". It may be postulated that intelligence drives through deficiency needs, and wisdom drives through growth needs. Detail postulate is given at end.

Knowledge is being considered the most important strategic resource, and the ability to create and apply it is the most important capability for building and sustaining competitive advantage. In the knowledge-based view individual may be observed as a vehicle for creating, integrating, storing, and applying knowledge.

Knowledge strategy may be the way in which individual balances own knowledge resources and knowledge processing capabilities with the knowledge required to create own performance in a manner superior to competitors. Knowledge and learning go hand in hand. Defending and growing a given knowledge position is most effectively accomplished by continual learning. The ability of an individual to learn, accumulate knowledge from own experiences, and reapply that knowledge is itself a skill or competence that, beyond the core competencies directly related to delivering own performance may provide strategic advantage.

An individual may be visualized as one who is running in the need cycle, like resource-based approach to strategic analysis of a firm. The need cycle may be defined as the fulfillment of all three (five) needs for a particular deficiency (growth) need achievement and again starting for another need. As for illustration take one's need fulfillment for achieving education, and then starting of need fulfillment of professional achievements. When there is inability to complete the need cycle for a specific need the hallucination need may come. Say a child starts receiving education for physical, safety, and social needs created by guardians. Later it is pupils' growth needs to have higher education. The end of achieving education depends on pupil's need. The end may be the start of professional engagement.

Knowledge in information sense is fundamental to human cognition and required both for theoretical speculation and practical sagacity [5]. The extraction of novel and unique patterns from generally available data and experience constitutes a source of new knowledge [Figure 1]. It reflects a reconfiguration of data from a diffused yet tacit state to a tacit but not-diffused state. Tacit knowledge 


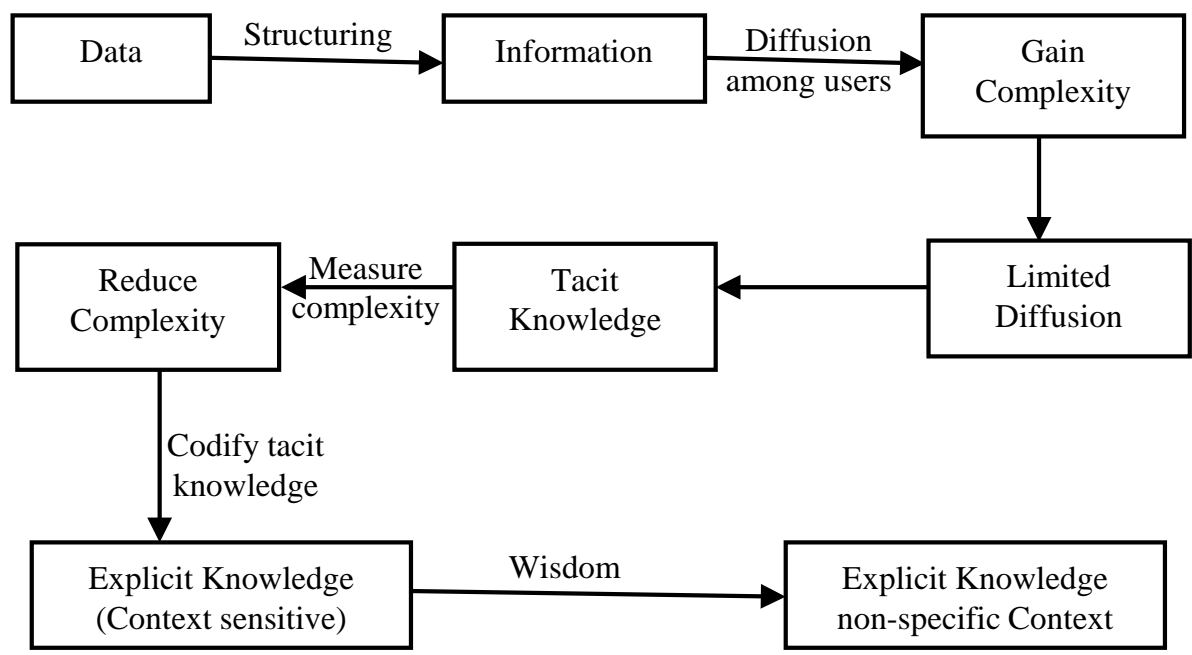

Figure 1. Reconfiguration of data from a diffused yet tacit state to a tacit but undiffused.

is knowledge that enters into the production of behaviours and/or the constitution of mental states but is not ordinarily accessible to consciousness. Tacit knowledge can be classified into three classes: 1) skills or expert performances, 2) cognitive competences like knowledge of language and 3) presuppositions or stances on many of our actions and behaviors committed to us. First and second kinds of tacit knowledge are domain specific. Third type or else tacit belief is generally applicable and plays across a diverse set of activities and domains. The ascription of tacit knowledge states to people is a theoretical move meant to explain behavior or cognitive operations. What makes ascriptions of tacit knowledge distinctive is the asymmetry between the richness of the ascribed content state and the relative poverty of the subjective experience corresponding to that state. The relation between the cognitive unconscious on one hand and conscious on the other is a complex operation to describe.

If (context free) wisdom is quantified on generating a structured context or a set of contexts, a wisdom module generates. Scanning of diffused wisdom module again generates not-diffused and codified data. This not-diffused and non-codified data or else collection of wisdom modules are to be codified for generation of explicit knowledge. Reference [17] has defined this codification, diffusion, absorption, and scanning process to run through codified, diffused, absorbed, and non-codified and not-diffused knowledge cycle as social learning cycle (SLC). On superimposition of data-information-knowledge-wisdom (DIKW) hierarchy [18]-Figure 2 with SLC we get intelligence improvement loop (IIL)-Figure 3 [8] [9] [10]. IIL is the collection of data, creation of information, crashing of information to knowledge quanta (KQ) and conceiving of KQ in long term memory (storing informative knowledge indefinitely) [18] on generation of explicit links to other KQs that are already in existence and subsequent generation of wisdom module to be re-collected as data.

The synthesizing procedure inside IIL may be a consequence of Generalizability 


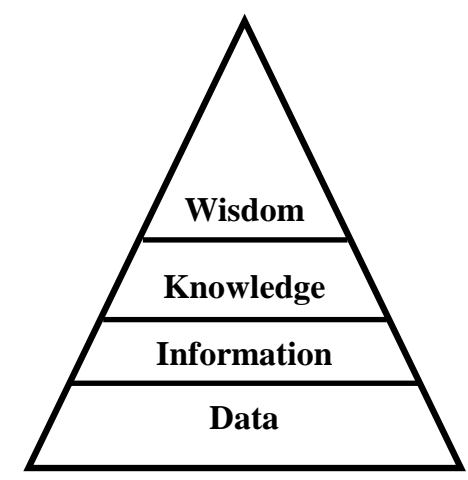

Figure 2. DIKW pyramid.

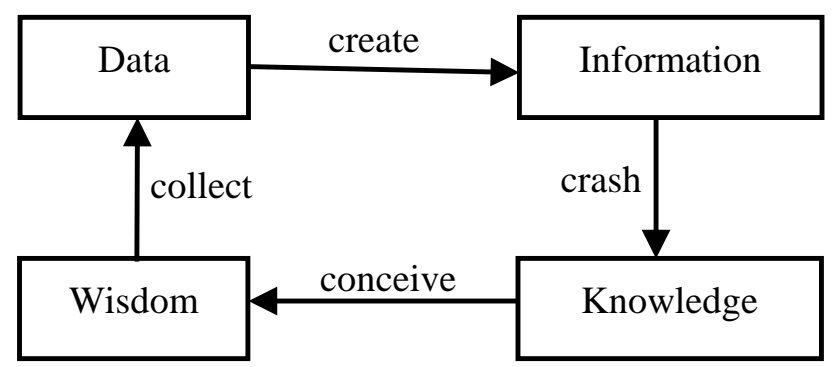

Figure 3. Intelligence improvement loop (IIL).

Thesis (GZT) [19]. As per GZT one cannot know that any given proposition $\mathbf{p}$ is true unless he/she also knows two other things. The first thing will be a certain more general proposition $\mathbf{q}$; $\mathbf{q}$ will not imply $\mathbf{p}$ but it will specify the condition under which proposition of a certain type is true. The second thing will be a proposition $\mathbf{r}$ that enables him/her to apply this general proposition to $\mathbf{p}$. In other words $\mathbf{r}$ will be a proposition to the effect that the first proposition $\mathbf{p}$ satisfies the condition specified in the second proposition q. But GZT implies that none know anything. To know $\mathbf{p}$, we need to know two other propositions $\mathbf{q}$ and $\mathbf{r}$. To know $\mathbf{q}$, and $\mathbf{r}$ we require four more propositions and so on. This stalemate is solved with need propositions. One's need (desire) is the priori that does not require other propositions to imply truth. We may refer Maslow's need hierarchy. At this point, we may define $H W$ as integration of HI with Desire. Intelligence is the $\mathbf{p}$ proposition of GZT, desire is the $\mathbf{q}$ proposition, and $\mathbf{r}$ is the integration operator or else wisdom (operator is a vital tool in QM). In other words it may be derived that wisdom re-configures intelligence as per desire. HW works not only in real time, but also thinks. Thinking may be defined as "analyzing", "synthesizing", and "imagining and goal setting". HW is looking for Recardian rent, i.e. desire to have something more.

"A foundation theory of justification must subscribe to the doctrine that at least some basic beliefs are fallible, or else embrace scepticism. The number of infallible beliefs is far too restricted to support our common sense claims to knowledge. Foundationalism is the doctrine that self-justified beliefs constitute the foundation of knowledge." [5] 
Illustration: Let us consider a normal newborn of age less than one month. What is her wisdom? She knows on feeling uneasiness she has to use her vocal cord (cry). This is priori. On feeling stomach pain (hungry) she uses vocal cord. She knows on cradling position if nipple is there she has to suck for milk (intelligence?). So even not hungry much, but on cradling position she seeks nipple it may be of her father (wisdom?).

Aristotle distinguished two kinds of intellectual virtue: philosophical and practical wisdom. Both kinds of wisdom are the result of training and not traits of character. Philosophical wisdom specifically is "intuitive reason combined with scientific knowledge", which is knowledge of best things, as opposed to what is to one's own advantage. According to fallibilist, justified mistakes are possible. For a fallibilist, wisdom can be understood in a way that seems more characteristics of what Aristotle thought of as moral virtue. That is, wisdom can be seen as a mean between the two extremes of believing without sufficient evidence and not believing with sufficient evidence-Table 1. As Hume put it, "A wise man, therefore, proportions his belief to the evidence". Hume thought that belief comes in degrees, so that the strength of belief would be proportional to the strength of evidence.

\section{Wisdom Differentiated from Intelligence}

Now let us have a qualitative differentiation between intelligence and wisdom with reference to Table 2 [8] [9] [10]. This table is outcome of superposition of

Table 1. Wisdom (WS) is a mean between two extremes of believing by Knowledge Quanta (KQ).

\begin{tabular}{llcl}
\hline \multicolumn{3}{c}{ Believing } \\
\hline \multirow{3}{*}{ Evidence } & & NO & YES \\
& NO & KQ & WS \\
& YES & WS & KQ \\
\hline
\end{tabular}

Table 2. Qualitative comparison between Intelligence and Wisdom.

\begin{tabular}{cc}
\hline INTELLIGENCE & WISDOM \\
\hline Certain & Acceptable \\
Evident & Counter balanced \\
Directly Evident & Self-presenting \\
Axiomatic & Priori \\
& Tends to confirm \\
Belief without doubt & Defeats \\
True belief de dicto & A set of concurrent propositions \\
Non-defectively Evident & True belief de re \\
Context sensitive & More probability than not
\end{tabular}


studies on [5] [19] [20] [21]. Wisdom may accept on reasoning among a number of choices. This reasoning is not looking only for "beyond reasonable doubt" choice. Beyond reasonable doubt (BRD) implies acceptance is more reasonable than withholding. BRD takes some presumptions that are to accept one proposition than to accept negation of that proposition. Intelligence uses BRD to be certain. If a person accepts that $\mathrm{p}$, then the person will be ready to affirm that $\mathbf{p}$ or to concede that $\mathrm{p}$ in appropriate circumstances and use $\mathrm{p}$ to justify other conclusions. Wisdom accepts but intelligence looks for certainty. Certainty means not only BRD but also acceptable among choices. Accepting something that is true does not suffice for knowledge but there is need for justification. Wisdom seeks doubts in acceptable proposition that is the first step towards invention or discovery. Wisdom reasons on seeking doubts to define certainty in time dependent frame. The doubt is outcome of the desire.

"Acceptance is the sort of mental state that has a specific sort of role, a functional role, in thought, inference, and action. When a person accepts that $\mathrm{p}$, he or she will draw certain inferences and perform certain actions assuming the truth of $\mathrm{p}$. Thus, if a person accepts that $\mathrm{p}$, then the person will be ready to affirm that $\mathbf{p}$ or to concede that $\mathbf{p}$ in the appropriate circumstances." [5]

In decision making when there is stalemate, intelligence selects "evident" (but not certain) among BRDs that are arising out from counter balancing propositions. Intelligence is the intersection of the "known truth" and the "tendency to avoid errors." In stalemate, Wisdom solves on describing "truth of inaction" (Inactions are those that have sufficient intelligence to involve for but not activated to involve) and "truth of prohibited action" (actions that are being prohibited by environment and/or system) properly with "desire." To describe wisdom uses GZT and IIL.

Wisdom may take different decisions on same situations but at different times. This may be through "self-presentation" that is a time dependent "evident" selection. In comparison, intelligence takes the "directly evident." Directly evident is the outcome of self-presentation of wisdom. One self-presentation gives truth value to other self-presentation. An axiom or basic truth has no other proposition prior to it. If one accepts an axiom it is certain. Whereas axiomatic may be axiom, may be acceptable but may not be certain. Any conjunction of axioms is itself an axiom. But any conjunction of axiomatic propositions for a subject may not be axiomatic.

A "priori" is the acceptance (but not certain) of an axiomatic proposition whose truth value is ascertained by another axiomatic proposition. In decision making intelligence works with axiomatic but wisdom may take priori. The truth value of priori grows with time that is on acquiring experience. But axiomatic is coming out of experience like posteriori knowledge. The decision making with priori calls for extra factor that added with posteriori becomes the key for the wisdom to unlock the counter balance. This factor we may call the "Desire".

When there is no certainty, acceptance, evident etc. the belief comes. Accep- 
tance involves evaluation in terms of the epistemic purpose. Belief does not involve evaluation in terms of their purposes. If belief is related to a purpose at all, it is just a by-product. Belief may result from the pursuit of some purpose, but it is defined in terms of any purpose.

Basic beliefs are basic because they cannot be false; their truth is guaranteed. With this initial guarantee of truth in basic beliefs, the next problem is how to extend this guarantee to other beliefs. Justification sufficient to ensure us knowledge must guarantee the truth of what we accept. The allegedly basic beliefs must stand in the appropriate probability relation to other beliefs for their justification. Basic beliefs guarantee their truth faces two problems, to guarantee their own truth and to guarantee the truth of other beliefs.

The belief that $\mathbf{p}$ is incorrigible for $\mathbf{S}$ if and only if 1 ) it is logically necessary that if $\mathbf{S}$ believes that $\mathbf{p}$, then it is true that $\mathbf{p}$ (infallibility condition) and 2 ) it is logically necessary that if it is true that $\mathrm{p}$, then $\mathbf{S}$ believes that $\mathrm{p}$ (irresistibility condition).

Whatever one can believe as a result of introspection, one can instead believe as a result of inference, and the inference can be based on false premises. Fallibility infects almost all our beliefs. The attempt to extend the guarantee of truth from basic to non-basic beliefs by undertaking to reduce the content of the latter to the contents of collections or conjunctions of the former is equally unsuccessful.

No belief can have justification which guarantees the truth of the belief, or that no beliefs are certain. The number of infallible beliefs is far too restricted to support our common sense claims to knowledge.

To work with belief, intelligence uses "belief without doubt" and "true belief de dicto" in a context sensitive manner. Belief without doubt has the character that is not negated with other acceptable propositions. The "true belief de dicto" is the acceptance of a "state of affairs" that occurs. The "true belief de re" is the attribution of one with a property that it already owns but not attributed earlier by the attributer. As for illustration; in an office one has daredevil critical problem solving capability. But supervisor and parallels do not weigh her, instead neglect her. When there is a requirement of critical problem solving she is being pushed alone and others go in background to promote her decisions and tactics. After the solution she is not appraised.

When intelligence works with beliefs, wisdom calls for tends to confirm, defeats, a set of concurrent propositions, and "true belief de re".

An e tends to confirm $\mathbf{h}$ : Necessarily, for every $\mathbf{S}$, if either 1$)$ e is evident for $\mathbf{S}$ and such that everything that is evident for $\mathbf{S}$ is entailed by e or 2) e is indirectly evident for $\mathbf{S}$ and such that everything that is indirectly evident for $\mathbf{S}$ is entailed by $\mathbf{e}$, then $\mathbf{h}$ has some presumption in its favor for $\mathbf{S}$. The expression " $\mathrm{e}$ is indirectly evident for $\mathbf{S}$ " may here be taken to abbreviate " $h$ is evident for $\mathbf{S}$ but neither directly evident nor a priori for $\mathbf{S}$ ". An $\mathbf{e}$ entails $\mathbf{h}$ if $\mathbf{e}$ is necessarily such that a) if it obtains/occurs/takes place, $\mathbf{h}$ obtains and b) whoever accepts it accepts $\mathbf{h}$. 
An $\mathbf{r}$ defeats the confirmation that $\mathbf{e}$ tends to provide for $\mathbf{h}$ if 1 ) e tends to confirm $\mathbf{h}$, and 2) the conjunction, $\mathbf{e}$ and $\mathbf{r}$, does not tend to confirm $\mathbf{h}$.

A "set of concurrent propositions" is a set of two or more propositions each of which is such that the conjunction of all the others tends to confirm it and is logically independent of it.

$\mathrm{A} \mathbf{h}$ is "non-defectively evident" for $\mathbf{S}$ if either $\mathbf{h}$ is certain for $\mathbf{S}$, or $\mathbf{h}$ is evident for $\mathbf{S}$ and is entailed by a conjunction of propositions each having for $\mathbf{S}$ a basis which is not a basis of any false proposition for $\mathbf{S}$. An $\mathbf{e}$ is a basis of $\mathbf{h}$ for $\mathbf{S}$ : $\mathbf{e}$ is self-presenting for $\mathbf{S}$; and necessarily, if $\mathbf{e}$ is self-presenting for $\mathbf{S}$, then $\mathbf{h}$ is evident for $\mathbf{S}$.

$\mathrm{A} \mathbf{h}$ is in the absolute sense "more probable than not" for $\mathbf{S}$ if there is an $\mathbf{e}$ such that 1) e is known by $\mathbf{S}, 2$ ) e tends to confirm $\mathbf{h}$, and 3) there is no $\mathbf{r}$ such that $\mathbf{r}$ is known by $\mathbf{S}$ and the conjunction of $\mathbf{e}$ and $\mathbf{r}$ does not tend to confirm $\mathbf{h}$.

\section{Quantum Mechanical Postulates}

Here I am putting forth my quantum mechanical (QM) postulates for the possibilities of it to be a tool on coding human behavior and wisdom. QM is the description of the behaviour of matter and light in all details and in particular of the happening in the atomic scale. For human unconscious behavior is so unlike ordinary conscious experience, it is very difficult to get used to, and it appears peculiar and mysterious.

Newton thought that light was made up of particles, but then it was discovered that it behaves like wave. The quantum behavior of atomic objects (electron, proton, neutron, photon, and so on) is the same for all, they are all "particle waves".

Because unconscious behavior is so unlike ordinary conscious experiences, it is very difficult to get used to, and it appears peculiar and mysterious to everyone. This paper is presenting nine postulates about the human behavior in presence of its dual nature as need and desire. Needs and desire are two aspects of human behavior, like particle-wave duality of sub-atomic particles.

As for illustration, one needs food to satisfy physical need of being hungry. Same person desires food of a posh restaurant to fulfill some other type of needs (say, safety, social and/or esteem) but not physical need of being hungry. So measuring physical need of hunger, the desire of hunger cannot be measured, unless some other factors are included. Again the measure of desire for hunger cannot let the measurement for need of hunger (one habituated with posh restaurant may be in need to take food from street vendor). In this way probabilistic measurement should come. Thus we get the uncertainty in simultaneous measurement of need and desire. This uncertainty is giving the possibility of QM description of human behavior and thus human wisdom. This may lead to creation of artificial wisdom. QM will measure behavior variability as amplitude that in turn will give the probability of occurrence. Different amplitudes are to be compared for the realization of probability of occurrence of a particular beha- 
vior.

Now I am putting my postulates towards possibility of quantum mechanical (QM) interpretation of human wisdom. These are just the preliminaries. A lot of future researches are in need (in desire to crack the artificial wisdom). These are an outcome of superposition of thoughts described above and that of reference [22].

\section{Postulate 1}

Wisdom $=$ Truth $=$ Intelligence + Desire;

Truth $=$ Truth of Action + Truth of Inaction + Truth of Prohibited Action;

Desire $=$ Need - Knowledge of Self - Unbiased Reasoning.

\section{Postulate 2}

Hallucination Need (HN) is defined as wandering of mind around unreal, acting with attachments, having deluded mind with egoism, performing as foolish follower. Each of three components of "desire" has embedded HN.

Each person has simultaneous attachments with a number of organizations, and/or institutions; like family, society, professional, educational, health care etc. The level and strength of these attachments are messy, and varying person to person, organization to organization. These attachments may be treated as packets or else quanta of knowledge/intelligence. The need dependent interaction of these quanta and interferences among them, may be analyzed quantum mechanically to have artificial wisdom.

\section{Postulate 3}

Each of five needs, "knowledge of self", and "unbiased reasoning" have two components. First is real and second is imaginary or else hallucination (HN). As for illustration, the physical need (PN) has two components the real and the imaginary or else hallucination physical need. For QM representation each need is represented with a complex number $(a+\mathrm{i} b)$, whose real part (a) relates to probability of the real need and imaginary part $(b)$ relates to probability of imaginary or hallucination need.

A set up that is acceptable may be termed as "a state of affairs" (a vector-in QM). Desire and its components may be expressed as state of affairs (as ket vector notation of QM).

Physical Need $(\mathrm{PN})=|\mathrm{PN}>=| a_{1}+\mathrm{i} b_{1}>$;

Safety Need $(\mathrm{SN})=|\mathrm{SN}\rangle=\left|a_{2}+\mathrm{i} b_{2}\right\rangle$;

Social Need $(\mathrm{CN})=|\mathrm{CN}\rangle=\left|a_{3}+\mathrm{i} b_{3}\right\rangle$;

Esteem Need $(\mathrm{EN})=|\mathrm{EN}>=| a_{4}+\mathrm{i} b_{4}>$;

Self-Actualization Need $(\mathrm{AN})=|\mathrm{AN}\rangle=\mid a_{5}+\mathrm{i} b_{5}>$;

Knowledge of Self $(\mathrm{KS})=|\mathrm{KS}\rangle=\mid a_{6}+\mathrm{i} b_{6}>$;

Unbiased Reasoning (UR) $=|\mathrm{UR}\rangle=\left|a_{7}+\mathrm{i} b_{7}\right\rangle$.

\section{Postulate 4}

Desire (DS) is taken as combination of (superposition in QM) needs, knowledge of self (KS) and unbiased reasoning (UR). This sum is to be interpreted as simultaneous occurrence of all states. 


$$
|\mathrm{DS}\rangle=|\mathrm{PN}>+| \mathrm{SN}>+|\mathrm{CN}\rangle+|\mathrm{EN}>+| \mathrm{AN}\rangle+|\mathrm{KS}\rangle+|\mathrm{UR}\rangle=\left|a_{8}+\mathrm{i} b_{8}\right\rangle ;
$$

Here $a_{m}$, and $b_{n}$ (where $m, n=1,2,3, \ldots$ ) are real numbers and $a_{m}^{2}+b_{n}^{2}=1$; $\mathrm{i}=\sqrt{-1} ; \mathrm{i} \cdot \mathrm{i}=\sqrt{-1} \cdot \sqrt{-1}=-1 ; \mathrm{i} \cdot \mathrm{i} \cdot \mathrm{i}=(\mathrm{i} \cdot \mathrm{i}) \cdot \mathrm{i}=(-1) \cdot \mathrm{i}=-\mathrm{i} ;$ and so on. For imaginary number computation the rules of algebra come.

Probability of real part of a state of affairs $=a_{m}^{2}$

Probability of imaginary or hallucination part of a state of affairs $=b_{n}^{2}$

The notation $|\ldots\rangle$ is called the Ket vector and originated from the word BracKet; that gives another notation the Bra vector $<\ldots .$. . If the ket vector is $\mid a_{m}+$ $\mathrm{i} b_{n}>$ the bra vector will be $<a_{m}-\mathrm{i} b_{n} \mid$. Bra and Ket vectors are conjugate to each other. As per Dirac [1] bra and ket vectors are conjugate imaginary which cannot be split into real and imaginary parts. This is in contrast to common complex number system that is termed by Dirac as conjugate complex.

Illustration: This is a thought illustration like thought experiment. We are hypothesising that need is manifestation of either No Knowledge (NOK), or Yes Knowledge (YSK) or Withhold Knowledge (WHK).

\section{Hunger $>$ YSK $>$ Need Food}

No Hunger but Salivation on Aroma $>$ NOK > Imagination of tasty food [aroma may be artificial but not from any food] NOK may cause search for similar taste food in future.

Also no hunger but passing by fast food outlet may initiate a short break to enjoy taste. No Hunger but passing by a posh restaurant; financial condition and/or time may not favour for costly posh restaurant. So WHK comes for future possibility. Thus we get the need to have a meal in a posh restaurant. Now we are again postulating that "no knowledge" and "withhold knowledge" give rise to the hallucination need. Let for a specific situation one's physical need for food be defined as $\mid \mathrm{YSK}+\mathrm{i}(\mathrm{NOK}+\mathrm{WHK})>$. $(\mathrm{YSK})^{2}=$ probability of yes knowledge, and $(\mathrm{NOK}+\mathrm{WHK})^{2}=$ probability of hallucination/unconscious need. $(\mathrm{YSK})^{2}+(\mathrm{NOK}+\mathrm{WHK})^{2}=1$. When this will occur simultaneously with other needs the desire comes. As for illustration, say an individual had regular habit to take fast food as evening snacks from street side food stall, and occasionally from posh restaurant as change of taste. But in widespread lock-down due to epidemic no street side food vendor was available, and also posh restaurants were only delivering at home. The person had no such financial condition to avail food of posh restaurant at home regularly. Now the desire and hallucination need come into work.

In this representation I am using one dimensional QM representation. But it may be represented more efficiently using quantum bits in Bloch Sphere that gives flexibility of matrix representation of probability using conjugate imaginary number. These states of affairs (vectors in QM) will be operated by operators to have transformation to other state of affairs. As for illustration, think one's state of affairs before and after an examination, where examination is the operator.

\section{Postulate 5}


Thinking may be defined as disturbance in desire that tries to rearrange needs with/without a net change in behavior. The rearrangement requires efficient "analysis", "synthesizing", and "imagining and goal setting."

Thinking transfers needs from one domain to others; say from social domain to esteem domain. It is desire function that moves needs on applications of operators (intelligence/wisdom). This movement of needs may be categorized in two classes-direct (longitudinal) and indirect (transverse). Direct transfer of need comes on fulfilling of one lower level need and moving up for higher level need; on satisfaction of physical need the safety need come and so on. Direct movement may also describe routine jobs. Indirect transfer occurs on activation of desire with wisdom operators. Illustration: on the course of achieving safety needs social need comes that again on the course of selecting leader generates esteem need that again call for safety need and so on. The action of operators may be like the Hawthorne effect [18] that refers to a type of reactivity in which individuals modify an aspect of their behavior in response to their awareness of being observed. On this affection one may change owns desired target from deficiency to growth, or from growth to deficiency.

\section{Postulate 6}

Thinking and consequent human behavior may be accounted as manifestation of socio-biological aspects and desire. The randomness of human behavior may be coping with probabilistic outcome of intelligence and/or wisdom operators on desire and socio-biological functions. Socio-biological function is to be defined for. The desire and socio-biological functions are to be elaborated statistically on statistical observations of human groups. Different entries in Table 2 should be taken as operators that work on function to output another function with change state.

\section{Postulate 7}

Need cycle with only deficiency needs may be called as intelligence operated system with zero hallucination need. Need cycle with deficiency and/or growth needs with hallucination components may be called as wisdom operated system.

\section{Postulate 8}

Need-Desire Uncertainty: It is impossible to measure needs individually without interferences among them, when ascertaining the desire that is framed out from those needs.

\section{Postulate 9}

Mind or else Wisdom Space is defined by five needs (physical, safety, social, esteem, and self-actualization) as dimensions, where each need space is defined as per postulate-1. Desire is combination of needs to achieve a particular need. Here we are considering multi-dimensional quantum space.

Neither the need view nor the desire viewpoint is correct. Both would be approximate. Through QM we are giving amplitude to every event that can occur. If the event involves the realization that one person in one specific need domain, we can give the amplitude to find that person at different needs at different times. The probability of finding the person in a specific need is then propor- 
tional to the absolute square of the amplitude. In general, the amplitude to find a person in different desires at different times varies with need and time. This amplitude may be taken as a variable that varies sinusoidally in need space and time like $\mathrm{e}^{\mathrm{i}(\omega t-k \cdot r)}$, where $\omega$ may be taken as angular frequency of specific need/desire variation with respect to time. But how we could assume that it varies sinusoidally with angular frequency. This requires further research. But it could be assumed that the variation of need is proportional to the need itself. So we could define an exponential function. Thought Illustration: Let in a courier office there be four delivery persons each one has one specific area of delivery location. They are coming on week days in the office take the job and go for delivery of items. This may be termed as horizontal activities. There may some other factors, like bad weather, family problem, leave of absence of one person loads other with extra job to cover the areas earmarked for that person. These are interference with horizontal activities, and may be called as vertical activities. The vertical activities burden horizontal activities to run smooth. Also horizontal activities are not in straight line. They also vary like number of items, weight and volume of items, spreading over the delivery addresses, etc.

On addition of vertical needs (scalar quantity) with horizontal needs (scalar quantity) we may get desire (vector quantity varying sinusoidally). This desire is an exponential function that varies with itself. Vertical as well or not horizontal components when coming into effect there must be some time difference of occurrence. This difference we may call phase difference, or else " $k r$ " term inside bracket of $\mathrm{e}^{\mathrm{i}(\omega t-k \cdot r)}$. Phase difference is the starting angle of counting of $2 \pi$ radian travel with respect to time of occurrence of main or first event.

These horizontal and vertical components may be represented by a standing wave as in Figure 4. This standing wave or else wave packet may also be defined by single beat note that is formed as addition of a number of sine waves with litthe variation in frequencies. The wave packet has zero amplitude everywhere except for a single localized region in space, over a region of width $\Delta x$. It may be taken as that individuals with common desires with little variations around a central objective may be called as traveling in a wave packet. This standing wave pattern could be analyzed quantum mechanically to have mathematical formula for human wisdom.

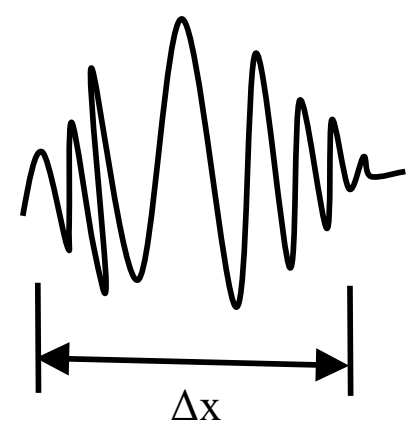

Figure 4. Standing wave. 
QM is characterized by measurements in intrinsic randomness, inference, and entanglement. The behavior of four delivery persons described above may have these. Intrinsic randomness may come into effect when one may try to complete assigned jobs in hurry, while one other may withhold some for next day. Inference phenomenon may come if one person on observation of hurriedly job completion, and withholding of jobs by other, may withhold one day's job for next day to be completed in hurry, and utilizes the free day with other engagement. Entanglement may come if this third person when utilizing the free day, may search for same type of job withholding and completion in hurry, in the other engagement.

\section{Conclusion}

I am concluding here. There is need to have integrated research to define and measure the probability of real and imaginary parts, operators that change state of affairs, and states of affairs. Also a mind function like wave function is to be defined for. Operators should act on the mind function to have observable as output human creative behavior.

\section{Conflicts of Interest}

The author declares no conflicts of interest regarding the publication of this paper.

\section{References}

[1] Dirac, P.A.M. (1947) The Principles of Quantum Mechanics. 3rd Edition, Clarendon Press, Oxford.

[2] Steven, H. (2013) Quantum Physics for Dummies. John Wiley \& Sons, Inc., Hoboken. https://www.wiley.com

[3] Feynman, R., Leighton, R. and Sands, M. (2006) The Feynman Lectures on Physics. The Definitive Edition, Volume 3, Pearson, London.

[4] Cresser, J.D. (2005) Quantum Physics Notes. Macquarie University, Sydney.

[5] Lehrer, K. (2000) Theory of Knowledge. Westview Press, Boulder.

[6] Williams, D.H. and Shipley, G.P. (2021) Enhancing Artificial Intelligence with Indigenous Wisdom. Open Journal of Philosophy, 11, 43-58.

https://doi.org/10.4236/ojpp.2021.111005

[7] https://twitter.com/Adriza2005/status/1161298498660159488?t=Izn0gBhXkZ-0GMc $\mathrm{L} 1 \mathrm{kFcRw} \& \mathrm{~s}=08$

[8] Sarkar, A. (2005) Generate Organizational Wisdom to Survive in Market Turbulence. National Seminar on Management Challenges - The Road Ahead, Dhanbad, 4-5 February 2005, 223-240.

[9] Sarkar, A. (2012) A Perspective to the Artificial Wisdom: Possibility of Self-Programmable Artificial Intelligence for Human Like Intelligence in Robotics. INDICON2012, Kochi, 7-9 December 2012, 183-188.

[10] Sarkar, A. (2015) Unlocking the Quest for Artificial Wisdom as Integration of Artificial Intelligence with Desire. Science Research, 3, 79-88.

https://doi.org/10.11648/j.sr.20150303.16 
[11] Prahalad, C.K. and Gary, H. (1990) The Core Competence of the Corporation. In: Zack, M.H., Ed., Knowledge and Strategy, Butterworth-Heinemann, Oxford, 41-59. https://doi.org/10.1016/B978-0-7506-7088-3.50006-1

[12] Donaldson, T. and Preston, L.E. (1995) The Stakeholder Theory of the Corporation: Concepts, Evidence and Implication. Academy of Management Review, 20, 65-91. https://doi.org/10.5465/amr.1995.9503271992

[13] Pearce, J.A. (1982) The Company Mission as a Strategic Tool. Sloan Management Review, Spring, 15-24. https://doi.org/10.2307/41164964

[14] Zack, M.H. (1999) Knowledge and Strategy. Butterworth-Heinemann, Oxford.

[15] Grant, R.M. (1991) The Resource Based Theory of Competitive Advantage: Implication for Strategy Formulation. In: Zack, M.H., Ed., Knowledge and Strategy, Butterworth-Heinemann, Oxford, 3-23.

https://doi.org/10.1016/B978-0-7506-7088-3.50004-8

[16] Maslow, A.H. (1943) A Theory of Human Motivation. Psychological Review, 50, 370-396. https://doi.org/10.1037/h0054346

[17] Boisot, M. (1999) Is Your Firm a Creative Destroyer? Competitive Learning and Knowledge Flows in the Technological Strategies of Firms. In: Zack, M.H., Ed., Knowledge and Strategy, Butterworth-Heinemann, Oxford, 251-273. https://doi.org/10.1016/B978-0-7506-7088-3.50017-6

[18] https://wikipedia.com

[19] Chisholm, R.M. (1977) Theory Knowledge. Second Edition, Prentice-Hall Inc., Hoboken.

[20] Mattey, G.J. (2003) Lecture Notes, Lehrer's Theory of Knowledge. Second Edition, Chapter Two. https://hume.ucdavis.edu/phi102kl/tkch2.htm

[21] Mattey, G.J. (2001) Self-Trust and the Reasonableness of Acceptance. https://hume.ucdavis.edu/phi102kl/trust1.pdf https://doi.org/10.1007/978-94-010-0013-0 12

[22] The Bhagavad-Gita, a Hindu Religion's Sacred Philosophical Book. 